\title{
Numerical Solution of Allen - Cahn Equation by Adomian Decomposition Method
}

\author{
Abdulghafor M. A. Al-Rozbayani \\ abdulghafor_rozbayani@uomosul.edu.iq \\ Department of Mathematics, College of Computer Science and Mathematics \\ University of Mosul, IRAQ
}

Received on: 24/3/2013

Accepted on: 24/6/2013

\begin{abstract}
In this paper, Adomian Decomposition Method with Adomian polynomials are applied to solve Allen - Cahn equation with the initial condition only, also DuFortFrankel method is applied with the initial and boundary conditions. The numerical results that are obtained by the Adomian decomposition method have been compared with the exact solution of the equation shown that it is more efficient than the DuFortFrankel method, that is illustrated through the tables and Figures.
\end{abstract}

Keywords: Allen - Cahn (AC) equation, Adomian decomposition method (ADM), DuFort-Frankel method (DFM).

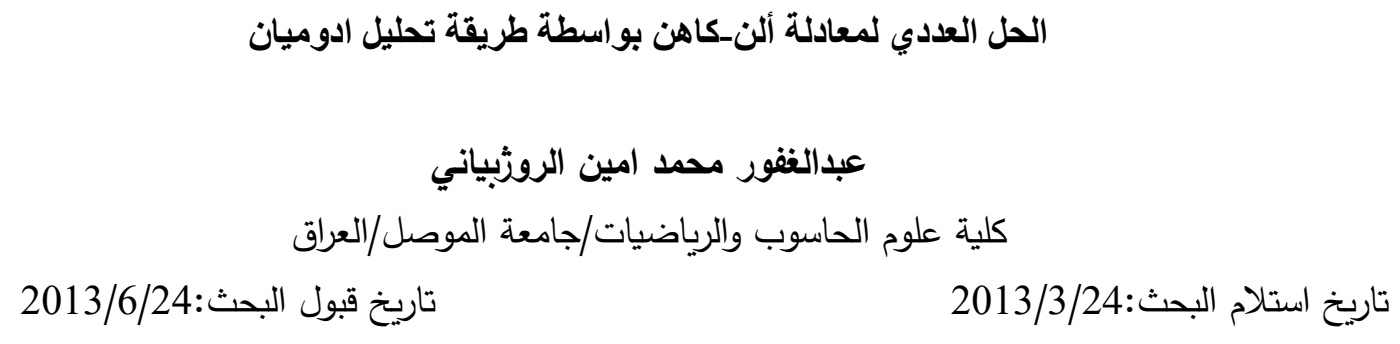

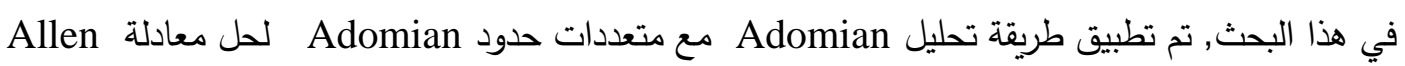

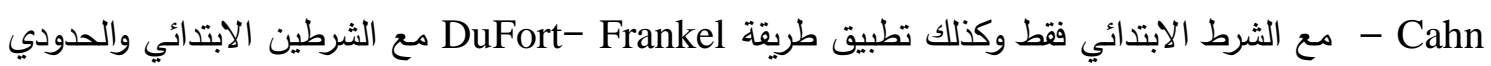

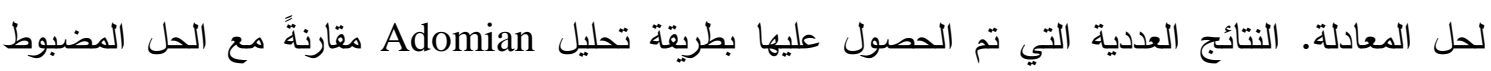
للمعادلة تبين بأنها أفضل من طريقة DuFort- Frankel كما هو موضح من خلال الجداول والأشكال. الكلمات المفتاحية: معادلة ألن-كاهن, طريقة تحليل ادوميان, طريقة دوفورت_فرانكل.

\section{Introduction}

Allen-Cahn equation is origin in the gradient theory of phase transitions [8], this equation is a model in which two distinct phases (represented by the values $u= \pm 1$ ) try to coexist in a domain $\Omega$ while minimizing their interaction which is proportional to the $(\mathrm{N}-1)$-dimension volume of the inter face.

Jeong- Whan Choi, et. al. in 2001, they used an unconditionally gradient table scheme for solving the Allen-Cahn equation representing a model for anti- phase domain coarsening in a binary mixture [19].

In 2011, Michal, et. al., introduced a numerical scheme allowing to perform computational studies of the anisotropic nonlocal Allen-Cahn equation [22]. 
The numerical solution of the generalized Allen-Cahn equation is used by Michal et. al. in 2004,[23] that proposed an algorithm for image segmentation, this technique is devoted to recovery of pattern boundaries from the original, possibly noisy image or signal.

Allen-Cahn equation, is a special type of non-linear partial differential equation, which arises as diffusion - convection equation in computational fluid dynamics or reaction - diffusion problem in material science. These equations are originally used to solve the phase transition problems, transformation of thermodynamic system from one phase to another due to an abrupt change in one or more physical properties. These equations describe the evolution of a diffuse phase boundary, concentrated in a small origin of size $\varepsilon$. It is also arising from phase transition in materials science [5,6].

Ali, et. al. in 2011, used some numerical methods for solving Allen-Cahn equation by different time stepping and space discretization methods with non-periodic boundary condition such as Chebyshov spectral method, fourth-order Runge-Kutta method, implicit-explicit scheme and finite difference scheme [6].

In the end of the years of the last century, Adomian [2,3,4] added a new method that solves differential equations, which is an efficient for analytic and numerical solution.

Ismail, et. al. in 2004, [16] studied the approximate solution for the Burger's Huxley and Burger's - Fisher equations and in [11] studied the numerical solutions of the Korteweg - De - Vries (KDV) and modified Korteweg - De - Vries (MKDV) equations, applied Adomian decomposition method.

Adomian decomposition method is also used to approximate the solution for the differential-algebraic equations (DAEs) systems [10,15].

Alabdulatifet. al. in 2007, [5] used Adomian decomposition method to find an analytic approximate solution for non-linear reaction diffusion system of Lotk Volterra type.

Javidi and Golbabai [18] used Adomian decomposition method for solving the linear and nonlinear parabolic equations is implemented with appropriate initial conditions and they show that is an efficient method.

Bokhari, et.al.in 2009, [9] shows that the Adomian series solution gives an excellent approximation to the exact solution for non-linear heat equation with temperature dependent thermal properties.

Ghoreishi, et. al. in 2010,[14] used ADM to solve non-linear wave - like equations with variable coefficients and they show that ADM is able to solve this type of equations without any need for dissertation, perturbation, transformation and linearization.

Abdelwahid, presented Adomian decomposition method is accuracy, applicability and simplicity to solve non-linear integral equation [1].

Space - time fractional telegraph equation is solved by Adomian decomposition method [13]. Cheniguel and Ayadi [12] presented the Adomian decomposition method for solving non homogenous heat equation with an initial condition and non local boundary conditions and they show that this technique is an efficient of the other classical methods.

Montazeri, applied Adomian decomposition method to solve a system of partial differential equations and show that the solutions, results are efficient [24].

Al-Khaled and Allan [7] are gave outlines a reliable strategy for solving nonlinear Volterra - Fredholmintegro - differential equations by (ADM) and from numerical examples they presented to illustrate - the accuracy of the method. 
Mamloukas, find an approximate solution of Burger's equation using the decomposition method [21].

In this research, we apply Adomian decomposition method and DuFort- Frankel method to Allen - Cahn equation [20]:

$u_{t}=\varepsilon u_{x x}+u-u^{3}, x \in[-1,1]$,

With initial condition

$u(x, 0)=0.53 x+0.47 \sin (-1.5 \pi x)$

And boundary conditions are

$u(-1, t)=-1, u(1, t)=1$

Then, the exact solution is

$u(x, t)=0.53 x+0.47 \sin (t-1.5 \pi x)$

\section{Analysis of TheAdomian Decomposition Method (ADM)}

In this section, we review the main steps of Adomian decomposition method on linear and non-linear parabolic partial differential equations with initial condition only, consider [30]:

$\frac{\partial u}{\partial t}=\frac{\partial^{2} u}{\partial x^{2}}+F(u)+g(x, t), \quad(\mathrm{x}, \mathrm{t}) \in[\mathrm{a}, \mathrm{b}] \times(0, \mathrm{~T}]$
$u(x, 0)=f(x), \ldots \ldots \ldots \ldots \ldots \ldots \ldots \ldots \ldots \ldots \ldots \ldots \ldots \ldots \ldots \ldots \ldots \ldots \ldots \ldots \ldots$

Where, $\mathrm{F}$ is a non-linear function of $\mathrm{u}$. We find the solution satisfying eqs. (4)-(5). Adomian decomposition method assumes that the unknown function $\mathrm{u}(\mathrm{x}, \mathrm{t})$ can be expressed by an infinite series of the form:

$u(x, t)=\sum_{n=0}^{\infty} u_{n}(x, t)$

And the non-linear operator $\mathrm{F}(\mathrm{u})$ can be decomposed by an infinite series of polynomial given by

$F(u)=\sum_{n=0}^{\infty} A_{n}\left(u_{0}, u_{1}, \ldots, u_{n}\right)$

Where, $\mathrm{A}_{\mathrm{n}}$ are the Adomian polynomials given by

$A_{n}=\frac{1}{n !} \frac{d^{n}}{d \lambda^{n}}\left[F\left(\sum_{\mathrm{k}=0}^{\infty} \lambda^{k} u_{k}\right)\right]_{\lambda=0}, \mathrm{n}=0,1,2 \ldots$

It is now well known in the literature, that these polynomials can be constructed for all classes of non-linearity according to algorithm set by Adomian $[3,4]$ and recently developed by an alternative approach in $[2,16,17,26,27,28,29]$.

Appling the decomposition method [16,17,18], eq.(1) can be written as

$L_{t} u=L_{x x} u+F(u)+g(x, t)$,

or

$L(u)=R(u)+N(u)+g$

Where, $L_{t}=\frac{\partial}{\partial t}$ and $\mathrm{L}_{\mathrm{xx}}=\frac{\partial^{2}}{\partial x^{2}}$. Assuming $\mathrm{L}$ is invertible, an $\mathrm{R}$ is a reminder operator and an $\mathrm{N}$ is a non-linear term. The inverse of operator $L_{t}$ exists and it can be taken as $L_{t}^{-1}()=.\int_{0}^{t}() d$.

Appling inverse operator on both sides of eq. (9) yields 
$\left.u(x, t)=u(x, 0)+L_{t}^{-1}\left(L_{x x} u\right)+L_{t}^{-1}(F u)\right)+L_{t}^{-1}(g(x, t))$

Then, using eqs.(6) and (7) it follows that

$\sum_{n=0}^{\infty} u_{n}(x, t)=f(x)+L_{t}^{-1}(g(x, t))+L_{t}^{-1}\left(\sum_{n=0}^{\infty}\left(u_{n}\right)_{x x}\right)+L_{t}^{-1}\left(\sum_{n=0}^{\infty} A_{n}\right)$

Where, $\mathrm{f}(\mathrm{x})=\mathrm{u}(\mathrm{x} .0)$. Therefore, determines the iterates in the following recursive way:

$u_{0}(x, t)=f(x)+L_{t}^{-1}(g(x, t))$,

$u_{n+1}(x, t)=L_{t}^{-1}\left(\left(u_{n}\right)_{x x}+A_{n}\right), \mathrm{n}=01,2, \ldots$

Then, when the non-linear function $\mathrm{F}(\mathrm{u})$ and by using eq.(8), Adomian

polynomials $[9,30]$ are given by:

$A_{0}=F\left(u_{0}\right)$,

$A_{1}=u_{1} F^{\prime}\left(u_{0}\right)$,

$A_{2}=u_{2} F^{\prime}\left(u_{0}\right)+\frac{1}{2 !} u_{1}^{2} F^{\prime \prime}\left(u_{0}\right)$,

$A_{3}=u_{3} F^{\prime}\left(u_{0}\right)+u_{1} u_{2} F^{\prime \prime}\left(u_{0}\right)+\frac{1}{3 !} u_{1}^{3} F^{\prime \prime \prime}\left(u_{0}\right)$,

Then, the solutions series are:

$u_{0}=f(x)+L^{-1} g(x, t)$,

$u_{1}=L^{-1} R\left(u_{0}\right)+L^{-1}\left(A_{0}\right)$,

$u_{2}=L^{-1} R\left(u_{1}\right)+L^{-1}\left(A_{1}\right)$,

$u_{3}=L^{-1} R\left(u_{2}\right)+L^{-1}\left(A_{2}\right)$,

$u_{1}=L^{-1} R\left(u_{n-1}\right)+L^{-1}\left(A_{n-1}\right)$.

Using the above recursive relation, we construct the solutions as:

$u(x, t)=\lim _{n \rightarrow \infty} \mathrm{u}_{\mathrm{n}}(x, t)$ Where, $\mathrm{u}_{\mathrm{n}}(x, t)=\sum_{\mathrm{k}=0}^{n-1} u_{k}(x, t), \mathrm{n} \geq 1$

It is interesting to note that, we obtain the series solution by using the initial condition only.

Then, the solution series of the equation that we get by ADM leads to the exact solution of $\mathrm{u}(\mathrm{x}, \mathrm{t})$ when $\mathrm{k}$ is an approach to infinity, i.e.

$u(x, t)=\lim _{n \rightarrow \infty} \mathrm{u}_{\mathrm{n}}(x, t)=\sum_{\mathrm{k}=0}^{\infty} u_{k}(x, t)$.

\section{Application of ADM to Allen-Cahn Equation}

In this section, we apply ADM to the Allen-Cahn eq. (1) with an initial condition in eq.(2).

Appling the inverse operator $L^{-1}$ on both sides of eq.(1) and using the initial condition, yields

$$
\sum_{n=0}^{\infty} u_{n}(x, t)=f(x)+\varepsilon L_{t}^{-1}\left(\sum_{n=0}^{\infty}\left(u_{n}\right)_{x x}\right)+L_{t}^{-1}\left(\sum_{n=0}^{\infty} u_{n}(x, t)\right)-L_{t}^{-1}\left(\sum_{n=0}^{\infty} A_{n}\right)
$$


Identifying the zeros component $u_{0}(x, t)$ by $f(x)$, the remaining components $n \geq 1$ can be determined by using recurrence relation $u_{0}(x, t)=f(x)$, $u_{n+1}(x, t)=\varepsilon L_{t}^{-1}\left(L_{x x} u_{n}\right)+L_{t}^{-1}\left(u_{n}\right)-L_{t}^{-1}\left(A_{n}\right), \mathrm{n} \geq 0$,

Where, $A_{n}$ are Adomian polynomial that represent the non-linear term $\left(u^{3}\right)$, then we can obtain from eq.(8) as follows:

$$
\begin{aligned}
& A_{0}=u_{0}^{3}, \\
& A_{1}=3 u_{0}^{2} u_{1}, \\
& A_{2}=3 u_{0}^{2} u_{2}+3 u_{0} u_{1}^{2}, \\
& A_{3}=3 u_{0}^{2} u_{3}+6 u_{0} u_{1} u_{2}+u_{1}^{3},
\end{aligned}
$$

Then, the solutions are obtained by using the initial condition only. Thus, we begin:

$$
\begin{aligned}
u_{0}(x, t)= & f(x), \\
u_{1}(x, t)= & \varepsilon L_{t}^{-1}\left(L_{x x} u_{0}\right)+L_{t}^{-1}\left(u_{0}\right)-L_{t}^{-1}\left(A_{0}\right), \\
u_{2}(x, t)= & \varepsilon L_{t}^{-1}\left(L_{x x} u_{1}\right)+L_{t}^{-1}\left(u_{1}\right)-L_{t}^{-1}\left(A_{1}\right), \\
\text { So } & \\
u_{0}(x, t)= & 0.53 x+0.47 \sin (-1.5 \pi x), \\
u_{1}(x, t)= & \varepsilon(-1.0575 \pi \sin (-1.5 \pi x) t)+0.53 x t+0.47 \sin (-1.5 \pi x) t \\
& -(0.53 x+0.47 \sin (-1.5 \pi x))^{3} t, \\
u_{2}(x, t)= & \varepsilon \frac{t^{2}}{2}\left(2.37935 \varepsilon \pi^{3} \sin (-1.5 \pi x)-1.0575 \pi^{2} \sin (-1.5 \pi x)\right. \\
& -(3(0.53 x+0.47 \sin (-1.5 \pi x)))^{2}(-1.0575 \pi \sin (-1.5 \pi x)) \\
& \left.+\left(6(0.53 x+0.47 \sin (-1.5 \pi x))(0.53-0.705 \pi \cos (-1.5 \pi x))^{2}\right)\right) \\
& +\frac{t^{2}}{2}((\varepsilon(-1.0575 \pi \sin (-1.5 \pi x))+0.53 x+0.47 \sin (-1.5 \pi x) \\
& \left.\left.-(0.53 x+0.47 \sin (-1.5 \pi x))^{3}\right)\left(1-3(0.53 x+0.47 \sin (-1.5 \pi x))^{2}\right)\right)
\end{aligned}
$$

\section{Application of DFM to Allen-Cahn Equation}

In this section, we apply DFM for Allen-Cahn eq. (1) with the initial condition in eq.(2) and boundary conditions in eq.(3). DFM is approximated the partial derivatives with respect to time $t$ and space $x$ with the second order accuracy through centered differencing formula, then eq.(1), by this method, leads to [25]:

$(1+2 r) u_{i}^{j+1}=(1-2 r) u_{i}^{j-1}+2 r\left(u_{i+1}^{j}+u_{i-1}^{j}\right)+2 k u_{i}^{j}-2 k\left(u_{i}^{j}\right)^{3}$

When, $x=i \Delta x, \mathrm{i}=0,1,2, \ldots, t=j \Delta t, \mathrm{j}=0,1,2, \ldots$

Where, $\Delta x=h, \Delta t=k$ and $r=\frac{\varepsilon k}{h^{2}}$. 


\section{Numerical Results}

The numerical solution of eq.(1) with eq.(2) by ADM is obtained when $u(x, t)=u_{0}(x, t)+u_{1}(x, t)+u_{2}(x, t)$, and the numerical solution of eq.(1) with eqs.(2) and (3) by DFM is obtained from eq.(14).

Then, the numerical results of (AC) equation by exact solution, ADM, and DFM are shown in table (1) with absolute errors when $-1 \leq x \leq 1, \varepsilon=0.001$ and $\mathrm{t}=0.1$, and in table (2) when $-1 \leq x \leq 1, \varepsilon=0.0001$ and $\mathrm{t}=0.1$. Also, the figures (1) and (2) shown the surface of the numerical solution $u(x, t)$ of (AC) equation when $-1 \leq x \leq 1,0 \leq t \leq 0.2$ in (a) the exact solution, in (b) ADM and in (c) DFM, then in (d) shown the comparison between the exact solution with ADM and DFM at $\varepsilon=0.001$ and $\varepsilon=0.0001$ respectively.

In order to show that numerically whether the Adomian decomposition for eq.(1) leads to a higher accuracy, we evaluate the approximate solution by using more than 3 terms approximations.

Table (1): Illustrated the solutions of the methods Exact, ADM and DFM and absolute errors between Exact and ADM and Exact and DFM, when $\mathbf{t}=\mathbf{0 . 1}$ and $\varepsilon=0.001$.

\begin{tabular}{|c|l|l|l|l|l|}
\hline $\mathrm{x}$ & \multicolumn{1}{|c|}{ Exact } & \multicolumn{1}{|c|}{ ADM } & \multicolumn{1}{|c|}{ DFM } & $\mid$ Exact-ADM $\mid$ & $\mid$ Exact-DFM $\mid$ \\
\hline-1.0 & -0.997651958 & -0.999707443 & -1.000000000 & 0.002055485 & 0.002348042 \\
\hline-0.8 & -0.736839381 & -0.735012753 & -0.734568681 & 0.001826628 & 0.002270700 \\
\hline-0.6 & -0.218112792 & -0.190444481 & -0.190497438 & 0.027668311 & 0.027615354 \\
\hline-0.4 & 0.218263837 & 0.257759265 & 0.257281675 & 0.039495427 & 0.039017837 \\
\hline-0.2 & 0.299918268 & 0.300268137 & 0.299856922 & 0.000349870 & 0.000061346 \\
\hline 0.0 & 0.046921706 & 0.000000000 & 0.000000000 & 0.046921706 & 0.046921706 \\
\hline 0.2 & -0.244758495 & -0.300268137 & -0.299856922 & 0.055509643 & 0.055098428 \\
\hline 0.4 & -0.247263046 & -0.257759265 & -0.257281675 & 0.010496218 & 0.010018628 \\
\hline 0.6 & 0.128862404 & 0.190444481 & 0.190497438 & 0.061582077 & 0.061635034 \\
\hline 0.8 & 0.660918467 & 0.735012753 & 0.734568681 & 0.074094287 & 0.073650215 \\
\hline 1.0 & 0.997651958 & 0.999707443 & 1.000000000 & 0.002055485 & 0.002348042 \\
\hline
\end{tabular}

Table (2): Illustrated the solutions of the methods Exact, ADM and DFM and absolute errors between Exact and ADM and Exact and DFM, when $\mathbf{t}=0.1$ and $\varepsilon=0.0001$.

\begin{tabular}{|c|l|l|l|l|l|}
\hline $\mathrm{x}$ & \multicolumn{1}{|c|}{ Exact } & \multicolumn{1}{|c|}{ ADM } & \multicolumn{1}{|c|}{ DFM } & $\mid$ Exact-ADM $\mid$ & $\mid$ Exact-DFM $\mid$ \\
\hline-1 & -0.997651958 & -0.999970711 & -1.000000000 & 0.002318753 & 0.002348042 \\
\hline-1 & -0.736839381 & -0.735096901 & -0.735019582 & 0.001742480 & 0.001819799 \\
\hline-1 & -0.218112792 & -0.190301469 & -0.190294914 & 0.027811323 & 0.027817877 \\
\hline-0 & 0.218263837 & 0.258088102 & 0.258018510 & 0.039824265 & 0.039754672 \\
\hline-0 & 0.299918268 & 0.300549962 & 0.300478938 & 0.000631694 & 0.000560671 \\
\hline 0 & 0.046921706 & 0.000000000 & 0.000000000 & 0.046921706 & 0.046921706 \\
\hline 0.2 & -0.244758495 & -0.300549962 & -0.300478938 & 0.055791468 & 0.055720444 \\
\hline 0.4 & -0.247263046 & -0.258088102 & -0.258018510 & 0.010825056 & 0.010755463 \\
\hline 0.6 & 0.128862404 & 0.190301469 & 0.190294914 & 0.061439065 & 0.061432511 \\
\hline 0.8 & 0.660918467 & 0.735096901 & 0.735019582 & 0.074178435 & 0.074101116 \\
\hline 1 & 0.997651958 & 0.999970711 & 1.000000000 & 0.002318753 & 0.002348042 \\
\hline
\end{tabular}


(a) Exact solution

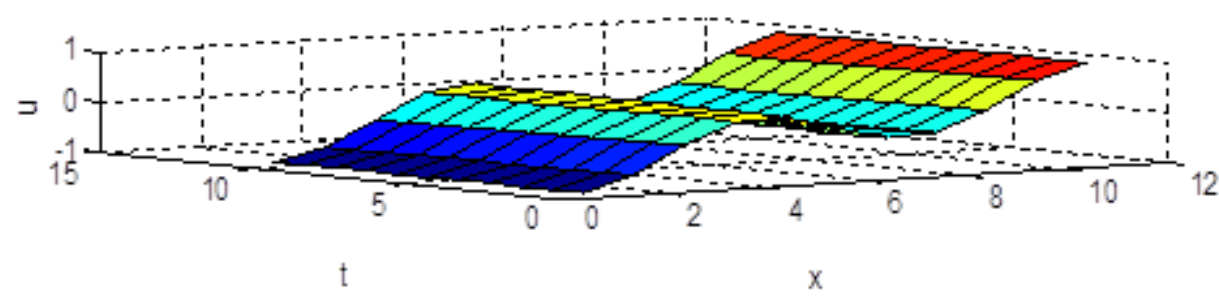

(b) ADM solution

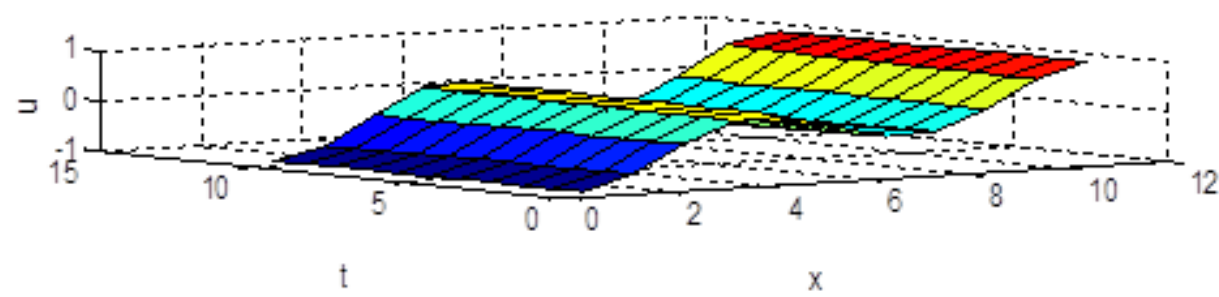

(c) DFM solution

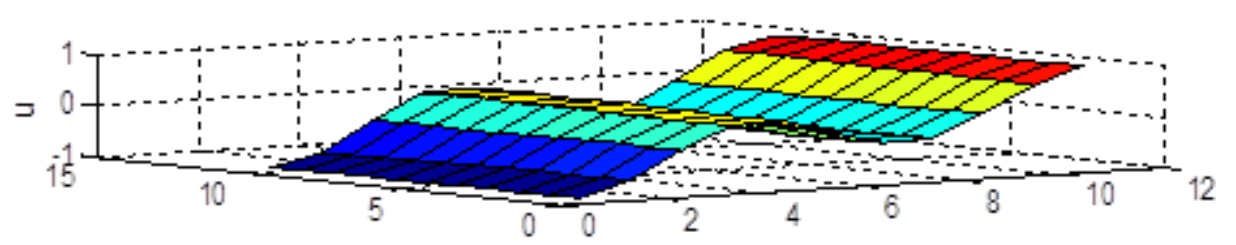

t

$\mathrm{x}$

(d) Comparis on Exact with ADM \& DFM at t $=0.1$ \& eps olon $=0.001$

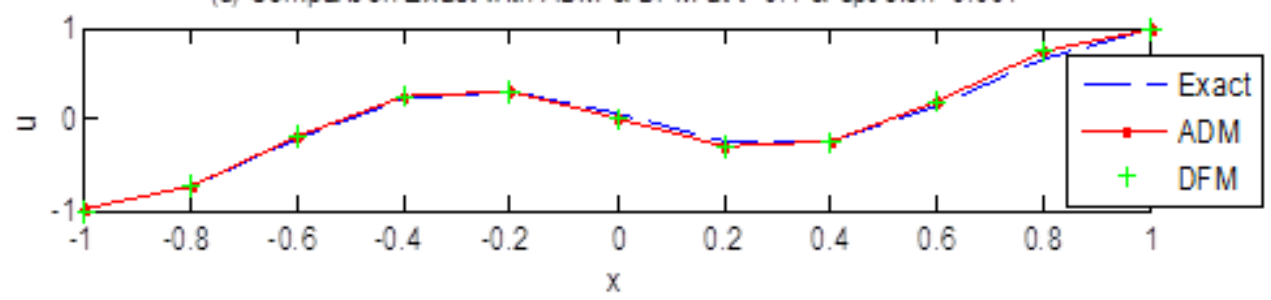

Figure (1)

(a)The numerical results for $u(x, t)$ are obtained by the exact solution with $\varepsilon=0.001$, (b)The numerical results for $u(x, t)$ are obtained by the ADM with $\varepsilon=0.001$, (c)The numerical results for $u(x, t)$ are obtained by the DFM with $\varepsilon=0.001$, (d) The comparison of the exact solution with the ADM and DFM with $\mathbf{t}=0.1$ and $\varepsilon=0.001$. 


\section{(a) Exact solution}

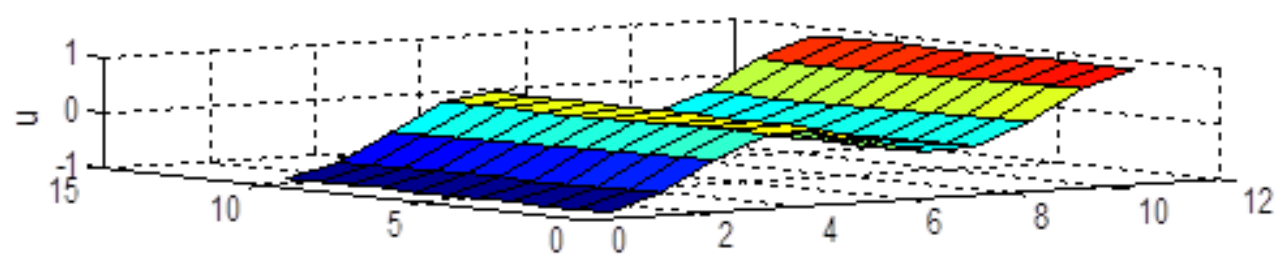

t $\mathrm{X}$

(b) ADM solution

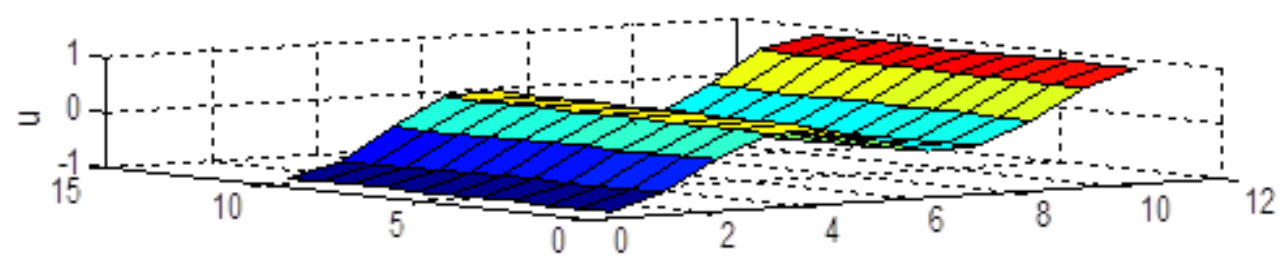

t $x$

(c) DFII solution

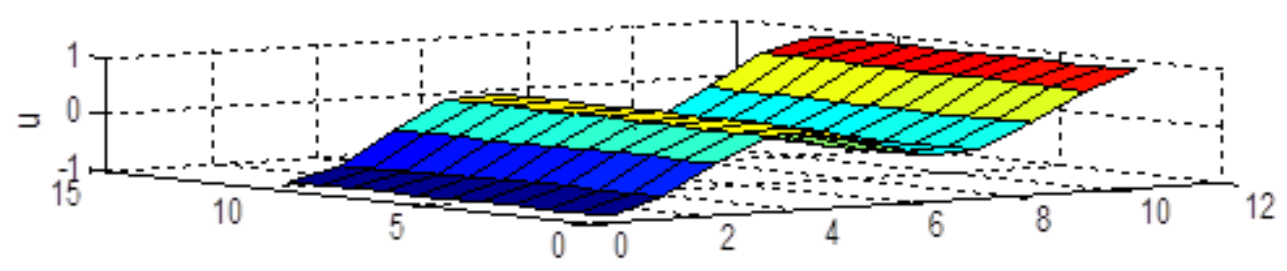

t $\mathrm{X}$

(d) Comparison Exact with ADII \& DFIM at $t=0.1$ \& epsolon $=0.0001$

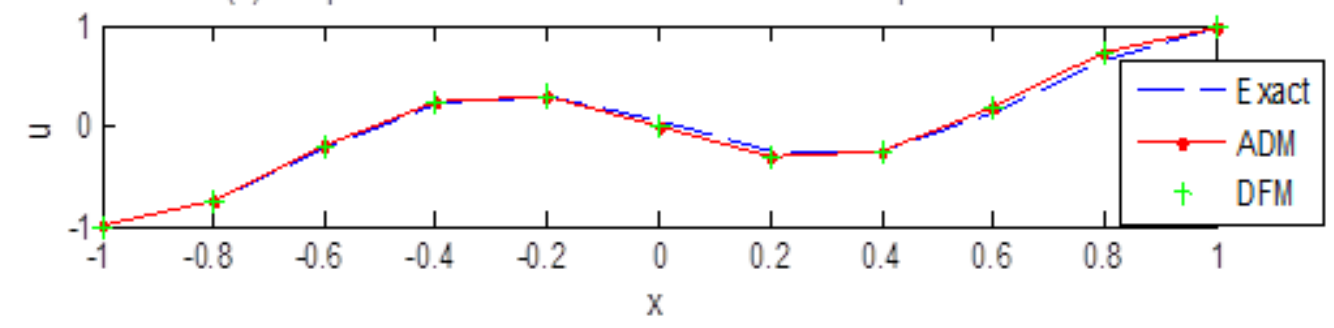

Figure (2)

(a) The numerical results for $\mathbf{u}(\mathbf{x}, \mathbf{t})$ are obtained by the exact solution with $\varepsilon=0.0001$,

(b) The numerical results for $\mathbf{u}(\mathbf{x}, \mathbf{t})$ are obtained by the ADM with $\varepsilon=0.0001$,

(c) The numerical results for $\mathbf{u}(\mathbf{x}, \mathbf{t})$ are obtained by the DFM with $\varepsilon=0.0001$, (d)The comparison of the exact solution with the ADM and DFM with $\mathrm{t}=0.1$ and $\varepsilon=0.0001$.

\section{Conclusion}

We note that the Adomian decomposition method is an efficient method when used Adomian polynomials to the treatment of the non-linear term in Allen - Cahn equation with convergence to the exact solution and small error. We see that the ADM has been applied directly without using bilinear forms. Also, we see that when we take added terms from the series solution, the ADM convergence is more efficient to the exact solution than the DFM. We used Matlab codes to obtain the solutions. 


\section{REFERENCES}

[1] Abdelwahid, F., (2010), " Adomian Decomposition Method Applied to Nonlinear Integral Equations", Alexandria Journal of Mathematics, Volume 1, Number 1, Pp. 11-18.

[2] Adomian, G., (1984), "new approach to nonlinear partial differential equations", J. Math. Anal. Appl., 102,420-434.

[3] Adomian, G., (1988), " A review of the decomposition method in applied mathematics", J. Math. Anal. Appl. 135, 501-544.

[4] Adomian, G., (1994), "Solving Frontier Problems of Physics: the Decomposition Method", Kluer Acad. Publ. Boston.

[5] Alabdulatif, M., Abdusalam and Fahmy, E. S., (2007), " Adomian Decomposition Method for Nonlinear Reaction Diffusion system of Lotka-Volterra Type", International Mathematical Forum, 2, no. 2, 88796.

[6] Ali, I, Ismail, S., Siddique, I. and Min-Allah, N., (2011), " Some Efficient Numerical Solutions of Allen-Cahn Equation with Non-periodic Boundary Conditions", International Journal of Nonlinear Science, Vol. 11, No. 3, Pp. 380-384.

[7] Al-Khaled, K. and Allan, F., (2005), " Decomposition Method for Solving Nonlinear Integro-Differential Equations", J. App. Math. \& Computing, Vol. 19, No. 1-2, Pp. 415-425.

[8] Allen, S. and Cahn, J.W., (1979),"A microscopic theory for anti phase boundary motion and its application to anti phase domain coarsening, Acta. Metall 27, 1084-1095.

[9] Bokhari, A. H., Mohmmaad, G., Mustafa, M. T. and Zaman, F. D., (2009), " Adomian Decomposition Method for a Nonlinear Heat Equation with Temperature Dependent Thermal Properties", Hindawi Publishing Crporation, Mathematical Problems in Engineering, Volume 2009, Pp. 1-12.

[10] Celik, E., Bayram, M. and Yeloğlu, T., (2006), " Solution of DifferentialAlgebraic Equations (DAEs)by Adomian Decomposition Method", International Journal Pure \& Applied Mathematical Sciences, Vol. 3, No. 1, Pp. 93-100.

[11] Chen, L.Q., (2005), "Phase - field methods for micro- structural evolution", Ann. Rev. Mater.Res. 32, 113-140.

[12] Cheniguel, A. and Ayadi, A., (2011), " Solving Non Homogeneous Heat Equation by the Adomian Decomposition Method", International Mathematical Forum, Vol. 6, no. 13, Pp. 639-649.

[13] Garg, M. and Sharma, A., (2011), " Solution of Space-Time Fractional Telegraph Equation by Adomian Decomposition Method", Journal of Inequalities and Special Functions, Volume 2, no. 1, Pp. 1-7.

[14] Goreishi, M., Ismail, A. I. B. Md. and Ali, N. H., (2010), " AdomianDecomposition Method (ADM) for Nonlinear Wave-Like 
Equations with Variable Coefficient", Applied Mathematical Sciences. Vol. 4, no. 49, 2431-2444.

Hosseini, M. M., (2006), " Adomian Decomposition Method for Solution of Differential-Algebraic Equations", Journal of Computational and Applied Mathematics, 197, 495-501.

Ismail, H. N. A., Raslan, K. and AbdRabboh, A., (2004), " Adomian Decomposition for Burger's-Huxly and Burger's-Fisher Equation", Applied Mathematics and Computation 159, 291-301.

Ismail, H. N. A., Raslan, K., and Salem, G. S. E., (2004), " Solitary Wave Solutions for the General KDV Equation by Adomian Decomposition Method", Applied Mathematics and Computation, 154, 17-29.

Javidi, M. and Golbabai, A., (2007), " Adomian Decomposition Method for Approximating the Solution of the Parabolic Equations" , Applied Mathematical Sciences, Vol. 1, no. 5, 219-225.

Jeon-Whan, C. , Hyun, L. and Junseok, K. , (2001),"An unconditionally gradient stable numerical method for solving the Allen-Cahn equation", Elsevier, Physical A 388, Pp. 1791-1803.

Kassam, A. and Trefethen, L. N., (2005), " Fourth-Order Time-Steeping for Stiff PDEs", SIAM J. SCI. COMPUT. Vol. 26, No. 4, Pp. 1214-1233.

Mamaloukas, C., (2002), " An Approximate Solution of Burger's Equation Using Adomian's Decomposition Method", The Conference of Applied Differential Geometry-General Relativity, Balkan Society of Geometers, Geometry Balkan Press, Pp. 88-98.

Michal, B. ,Praha, S.and Miyazaki, (2011),"Computational studies of Non-Local Anisotropic Allen-Cahn equation", J. MathematicaBoHEMicA, Vol. 136, No.4, Pp. 429-4437.

Michal, B. ,Vladimir, C. and Karol, M., (2004), "Geometrical image segmentation by Allen-Cahn equation", Journal of Applied Numerical Mathematics, Vol.51, Pp. 187-205.

Montazeri, R., (2012), " A Concrete Application of Adomian Decomposition Method Int. J. Contemp. Math. Sciences, Vol. 7, no. 24, Pp. 1185-1192.

Petrović, Z. and Stupar, S., (1996) " Computational Fluid Dynamics One", Mechanical Engineering Faculty, Belgrade.

Wazwaz, A. M., (1999), " The Modified Decomposition Method and Padé Approximants for Solving the Thomas-Fermi Equation", Appl. Math. Comput. 105, Pp. 11-19.

Wazwaz, A. M., (2000), " A new Algorithm for Calculating Adomian Polynomial for Nonlinear Operators", Appl. Math. Comput. 111, Pp. 5369.

Wazwaz, A. M., (2000), " Solitary Wave Solutions for the Modified KDV Equation by Adomian Decomposition Method, Int. J. Appl. Math., Vol. 3, no. 4, Pp. 361-368. 
Wazwaz, A. M., (2001), " Construction of Solitary Wave Solutions and [29] Rational Solutions for the KDV Equation by Adomian Decomposition Method", Chaos, Solitons Fractals 12, Pp. 2283-2293.

Wazwaz, A. M., (2009), " Partial Differential Equations and Solitary Wave Theory", Higher Education Press, Beijing and SpringerVerlagBerlinHeidelberg. 